\title{
Radiological imaging finding of calcified pseudoneoplasm of neural axis
}

\author{
Aishwerya Singh $M D^{1}$, Deepak Kumar $M D^{2}$, Umakant Prasad $M D^{3}$ \\ ${ }^{1,2,3}$ Associate Professor, Department of Radio-diagnosis, Indira Gandhi Institute of Medical Science, Patna, India \\ Date of submission: $24^{\text {th }}$ September $2020 \quad$ Date of acceptance: $12^{\text {th }}$ November $2020 \quad$ Date of publication: $1^{\text {st }}$ December 2020
}

\begin{abstract}
Calcified pseudoneoplasm of neural axis (CAPNON) is rare non-neoplastic non-inflammatory heavily calcified lesions of neural axis which can be intra-parenchymal or extra-axial and have been reported within brain and spinal cord with equal frequency. Here we describe unique radiological (CT \&MRI) Imaging finding of CAPNON in left hippocampus on CT scan and MRI.
\end{abstract}

Key words: Calcified pseudoneoplasm of neural axis (CAPNON), MRI, Temporal lobe

\section{Introduction}

Cinftip APNON is rare slow growing, non-neoplastic noninflammatory heavily calcified lesions of neural axis which can be intra-parenchymal or extra-axial and have been reported within brain and spinal cord with equal frequency. ${ }^{1}$ The etiology of the lesion is still unknown; however, investigators favor it to be a reactive rather than a neoplastic process. ${ }^{2}$ Patients with CAPNON are usually asymptomatic and found incidentally but sometimes they can present with a seizure or headache mainly due to mass effect or local irritation of adjacent tissue rather than local invasion because CAPNONs are usually considered to be benign non-infiltrative lesions. ${ }^{3}$ The most common

Access this article online
Website: https://www.nepjol.info/index.php/NJN
DOI: https://doi.org/10.3126/njn.v17i3.33124
HOW TO CITE
Singh A, Kumar D, Prasad U. Radiological imaging finding of
calcified pseudoneoplasm of neural axis. NJNS. 2020;17(3):41-44

'ORCID id: 0000-0003-2902-544X

${ }^{2}$ ORCID id: 0000-0002-5460-2707

${ }^{3}$ ORCID id: 0000-0003-3818-9323

\section{Address for correspondence:}

Dr Deepak Kumar

Room No- 251, Department of Radiodiagnosis,

Indira Gandhi Institute of Medical Science, Sheikhpura,

Patna-800014, Bihar

Phone: +91-9835284456

E-mail: 1999deepak@gmail.com

Copyright (C) 2020 Nepalese Society of Neurosurgeons (NESON)

ISSN: 1813-1948 (Print), 1813-1956 (Online) location is temporal region. This is a unique case report $\&$ we describing imaging findings of CAPNON in left hippocampus.

\section{Case Report}

We present a case of 23 year old female with refractory partial seizure of right side, admitted in neurology department of I.G.I.M.S., Patna. Patient came to Radiology department for CT scan.

On NCCT, amorphous calcification was seen at left hippocampus with subtle effacement of temporal horn of left lateral ventricle but no peri-lesional edema noted, then advised MRI for further characterization of lesion.

MRI performed on 1.5 Tesla system revealed a well defined T1W/T2W hypointense lesion which blooms on GRE* sequence and show enhancement after contrast administration (Fig- $2 \& 3$ ). No perilesional edema noted.

\section{Discussion}

The first intracranial calcifying pseudoneoplasm of neural axis was reported in 1978 by Rhodes and Davis ${ }^{1}$. Since then, 40 intracranial lesions have been found ${ }^{2}$. Calcified pseudoneoplasm of neural axis (CAPNON) is rare slow growing,non-neoplastic non-inflammatory heavily calcified lesions of neural axis. it can be intraparenchymal or extra-axial and have been reported within brain and spinal cord with equal frequency. ${ }^{3}$ They do not seem to have a predilection for sex, age, or central nervous system location ${ }^{1,4,5,6}$. The patient age ranges from 12 to 83 years. ${ }^{2}$ Most reported lesions have been extra-axial, but several intra-axial cases have also been reported. ${ }^{1}$ Patients with CAPNON are usually asymptomatic and found incidentally but sometimes they can present with a seizure and second most common symptom is headache/pain, 


\section{Singh et al}

likely due to focal compression or irritation of adjacent tissue. The radiological finding is typical, On NCCT, lesion appear well defined \& calcified intracranial mass. On MRI, Lesion is hypointense on T1W/T2W with no perilesional edema, bloom on GRE sequence \& show minimal to mild enhancement after contrast administration. CAPNON should be considered as close differential diagnosis of a heavily calcified lesion in neural axis in imaging. It is important to consider this entity to avoid aggressive surgical intervention. The close differential diagnosis for a heavily calcified lesion is Cavernoma, Oligodendroglioma, calcified Meningioma and calcified granuloma. The key distinguishing feature of CANON is uniform T1 and T2 hypointensity without solid enhancement. Calcified lesions with T2 hyperintensity or heterogenicity are more likely to be a calcified neoplasm and inconsistent with CAPNON. In our case, lesion appear uniform hypointense on $\mathrm{T} 2 \mathrm{~W}$ sequence. Calcified lesions with the typical popcorn (hyperintensity) appearance on T2 and peripheral hemosiderin ring are more consistent with cavernous malformation and easily distinguish it from CAPNON. Standard treatment is complete resection and is known to be curative. Due to benign behavior of lesion, no need of additional chemotherapy or radiotherapy. After complete resection of this lesion, the prognosis is typically favorable and complications tend to arise, if there is adhesions to adjacent arachnoid along the cranial nerves, blood vessels, or brainstem which rendering unsafe complete excision. ${ }^{3}$
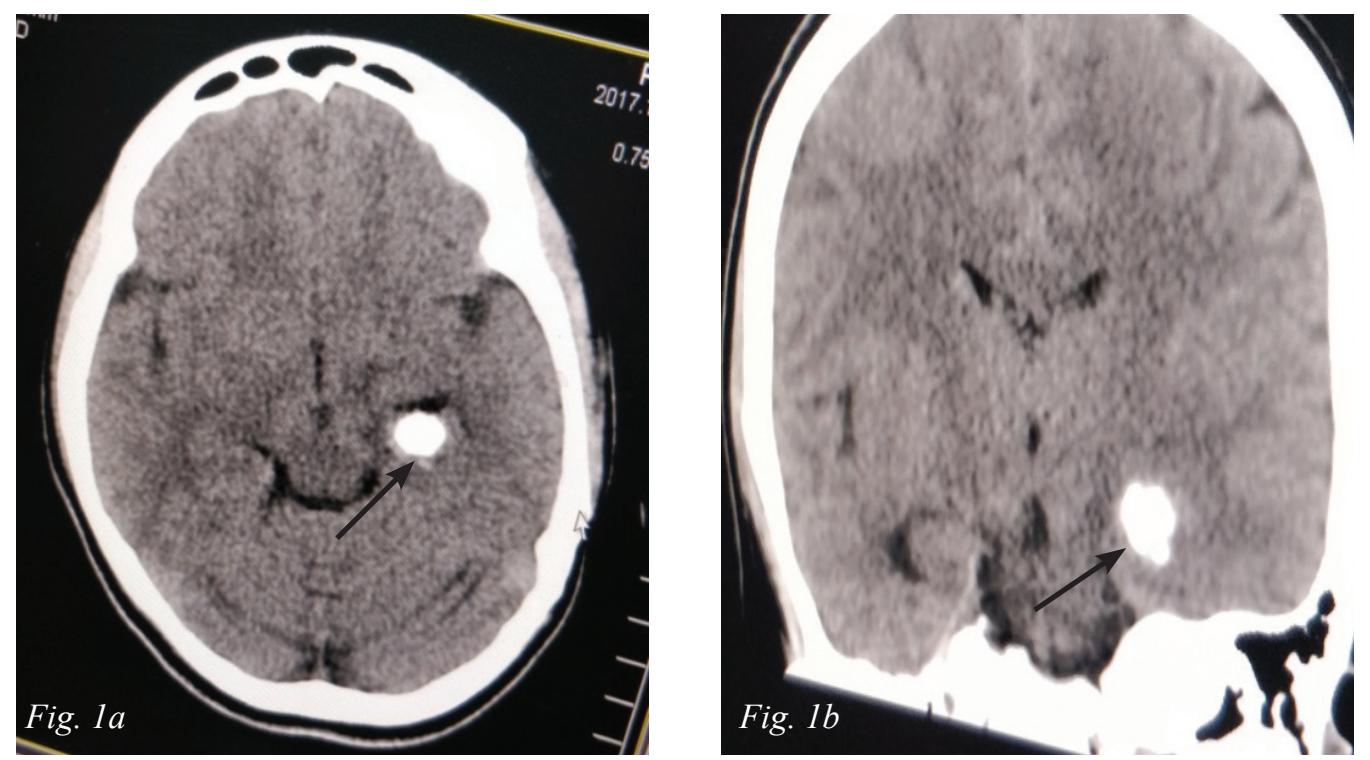

Figure la Axial \& 1 b coronal image of NCCT BRAIN showing amorphous calcification in left hippocampus. (Note no perilesional edema is visualized).
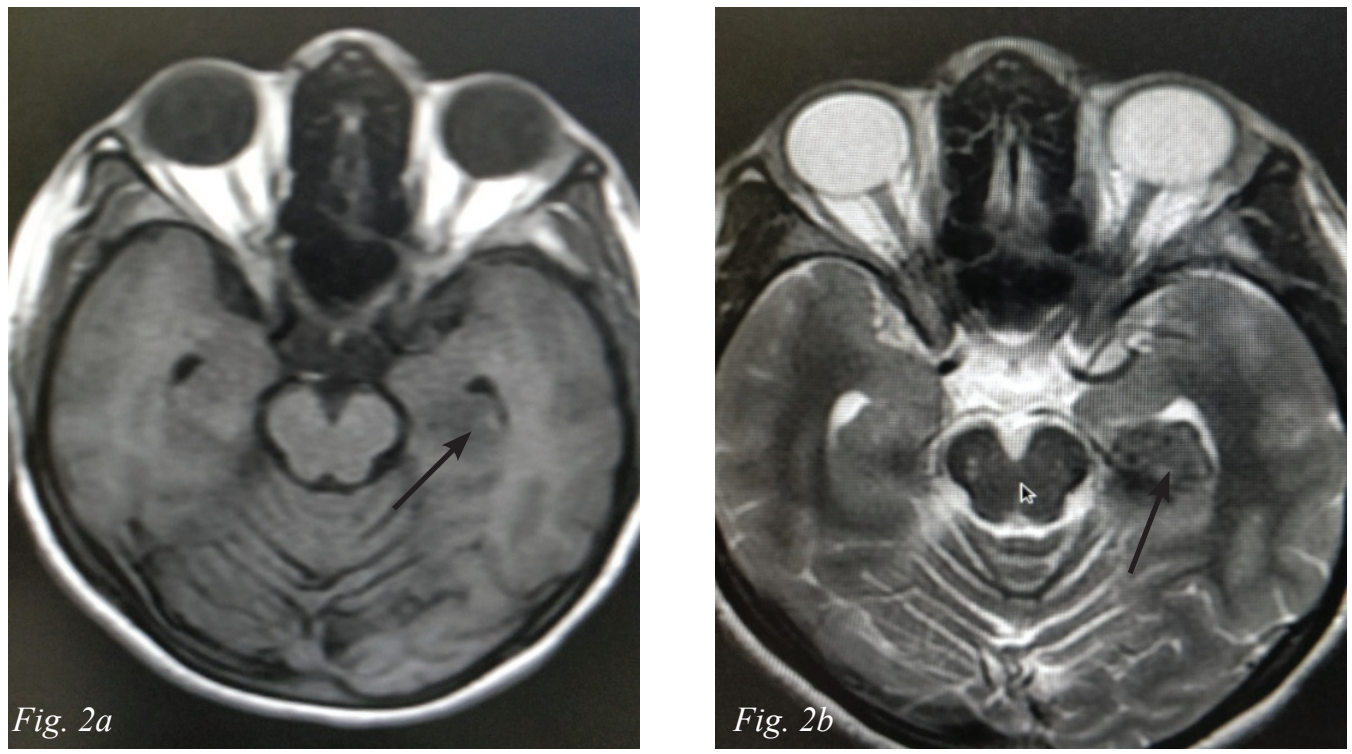

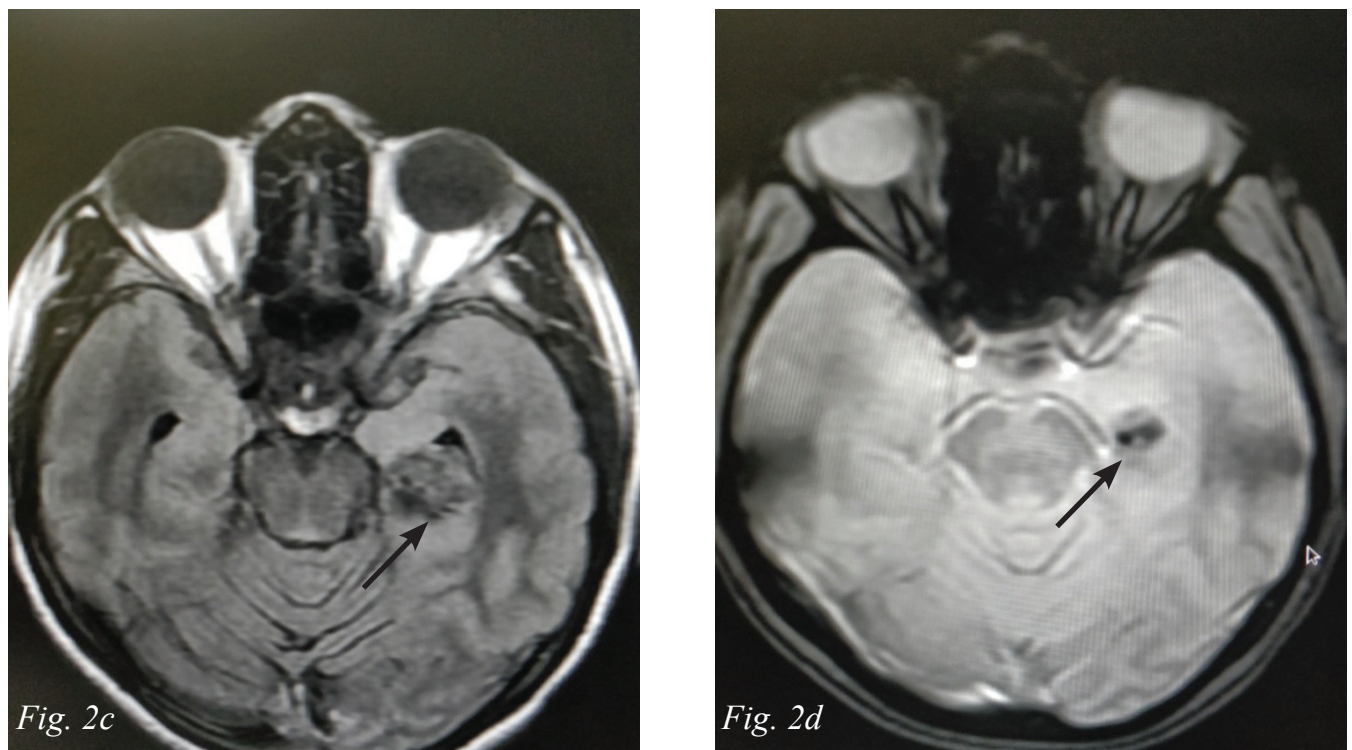

Figure 2: Axial MRI Images shows T1W (2a), T2W (2b), FLAIR (2c) hypointense lesion seen at left hippocampus (black arrow) which shows blooming on GRE*(2d).
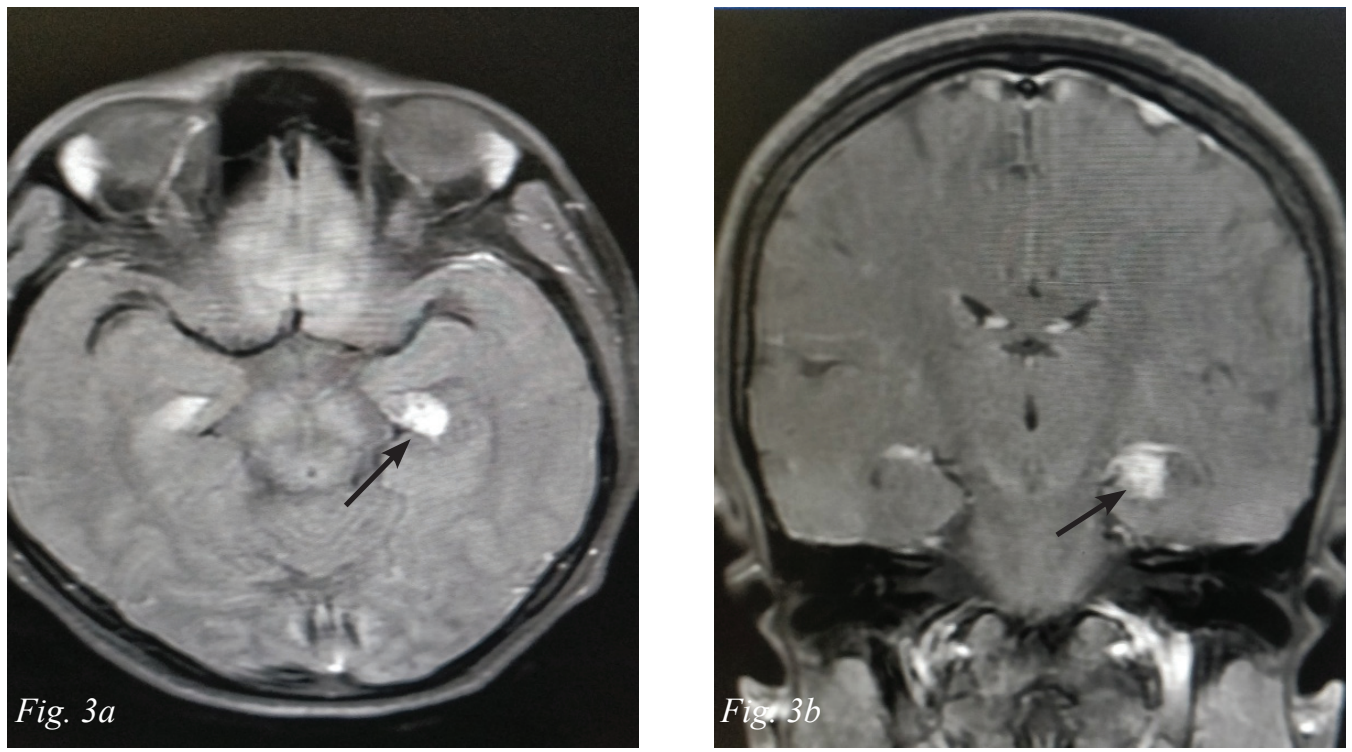

Figure 3: On gadolinium injection, contrast enhancement was visualized within lesion in the left hippocampus on axial (3a) and coronal (3b) images.

\section{Conclusion}

When a heavily calcified lesion is found on CT and lesion appear hypointense on T1-weighted and T2weighted MR image which blooms on GRE sequence and shows none to minimal post contrast enhancement and no perilesional edema, CAPNON should be considered as close differential diagnosis, to avoid aggressive diagnostic procedure.

Acknowledgement: The authors would like to thank patient, his family \& Department of Radiology of this institute.

\section{Conflict of Interest: None}

Source(s) of support: None

\section{References}

1. Rhodes RH, Davis RL. An unusual fibro-osseous component in intracranial lesions. Hum Pathol 1978; 9:309-19. https://doi.org/10.1016/S00468177(78)80088-4

2. Qian J, Rubio A, Powers JM, et al. Fibro-osseous lesions of the central nervous system: report of four cases and literature review. Am J Surg Pathol 1999; 


\section{Singh et al}

23:1270-75. https://doi.org/10.1097/00000478199910000-00013

3. Kerr EE, Borys E, Bobinski M, Shahlaie K: Posterior fossa calcifying pseudoneoplasm of the central nervous system. J Neurosurg. 2013, 118:896-902. https://doi.org/10.3171/2013.1.JNS121755

4. Garen PD, Powers JM, King JS, et al. Intracranial fibro-osseous lesion. Case report. J Neurosurg 1989;70:475-77.

https://doi.org/10.3171/
5. Jun C, Burdick B. An unusual fibro-osseous lesion of the brain. Case report. J Neurosurg 1984;60:1308-11. https://doi.org/10.3171/jns.1984.60.6.1308

6. Smith DM, Berry AD, 3rd. Unusual fibro-osseous lesion of the spinal cord with positive staining for glial fibrillary acidic protein and radiological progression: a case report. HumPathol 1994;25:83538. https://doi.org/10.1016/0046-8177(94)90256-9

jns.1989.70.3.0475 